\title{
AVALIAÇÃO DE DESCRITORES NA ANGIOLOGIA E CIRURGIA VASCULAR EM ARTIGOS PUBLICADOS EM DOIS PERIÓDICOS NACIONAIS ${ }^{1}$
}

\author{
Eymard Francisco Brito de Oliveira ${ }^{2}$ \\ Heriberto Brito de Oliveira ${ }^{3}$ \\ João Luiz Moreira Coutinho de Azevedo ${ }^{4}$ \\ Djalma José Fagundes ${ }^{5}$
}

\begin{abstract}
Oliveira EFB, Oliveira HB, Azevedo JLMC, Fagundes DJ. Descritores em ciências da saúde em artigos de periódicos, na área de angiologia e cirurgia vascular. Acta Cir Bras [serial online] 2003 Jan-Fev;18(1). Disponível em URL: http://www.scielo.br/acb.

RESUMO - A recuperação de referências na literatura biomédica está normatizada pelos Descritores em Ciências da Saúde (DeCS), contudo os autores de artigos científicos nem sempre obedecem ao regulamentado, o que causa dificuldade a localização da informação. Os autores avaliaram os Descritores em Ciências da Saúde em artigos de dois periódicos nacionais, na angiologia e cirurgia vascular no período de 1995 a 2000 em relação a adequada utilização dos descritores de acordo com a listagem do DeCS 2001 e do MeSH 1994. Foram estudados os descritores em 186 artigos publicados em 02 periódicos da especialidade. Foi observado que a maioria dos descritores empregados não estão de acordo com o DeCS 2001 e com o MeSH 1994. Concluiu-se que a indexação deva ser uma atividade dinâmica e que novos termos devem ser acrescentados para acompanhar o desenvolvimento da especialidade.Por outro lado, percebe-se uma desinformação por parte dos autores, que devem ser estimulados a utilizarem corretamente os descritores e a sugerirem a inclusão dos novos termos, como prevê a sistemática da indexação.
\end{abstract}

DESCRITORES - Descritores. Ciência da informação. Resumos e indexação. Catalogação, arquivamento.

\section{INTRODUÇÃO}

O grande número de informação gerado pelas publicações médicas leva a dificuldade de sua localização e sua apreciação ampla e segura.

A necessidade de classificar as informações para futuros levantamentos em estudos e pesquisas levou a criação de descritores, também conhecidos como unitermos ou palavras-chaves (key-words), tornando mais fácil o levantamento bibliográfico.

Os Descritores em Ciências da Saúde (DeCS), derivados do termo em língua inglesa Medical Subject Headings (MeSH) são publicados pela Biblioteca

1. Trabalho realizado no Programa de Pós Graduação em Técnica Operatória e Cirurgia Experimental (TOCE) da Universidade Federal de São Paulo - Escola Paulista de Medicina (UNIFESP - EPM).

2. Aluno do Curso de Fundamentação para a Docência e Pesquisa em Técnica Operatória e Cirurgia Experimental da UNIFESP EPM. Membro Efetivo da Sociedade Brasileira de Angiologia e Cirurgia Vascular. Mestre e Doutorando em Educação - Universidade Vale do Rio Verde de Três Corações - MG.

3. Médico Residente em Cirurgia Cardiovascular da Santa Casa de Misericórdia de Belo Horizonte - Faculdade de Ciências Médicas de Minas Gerais.

4. Professor Adjunto da Disciplina de Téc. Oper. e Cir. Exper.- UNIFESP - EPM.

5. Professor Adjunto da Disciplina de Téc. Oper. e Cir. Exper. e Coordenador do Programa de Pós-Graduação em TOCE - UNIFESP - EPM. 
Nacional de Medicina Norte Americana (U.S. National Library of Medicine), responsável pela base de dados Medline que é uma das mais completas e utilizadas na pesquisa bibliográfica na área das Ciências da Saúde.

Outras bases de dados como o Lilacs (Literatura Médica da América Latina e do Caribe) e Scielo (Scientific Eletronic Library Online) também disponíveis na Biblioteca Regional de Medicina (Bireme) usam a padronização dos DeCS.

É fundamental que os autores dos trabalhos científicos façam a escolha criteriosa e adequada destes unitermos, para que a indexação permita a recuperação de seus trabalhos, quando procurados pelos seus pares em uma pesquisa bibliográfica ${ }^{(1)}$.

Estudo dos descritores utilizados em 29 teses de mestrado em Tisiologia e Pneumologia do IDT-UFRJ (1990-1996), mostra que somente 14\% dos descritores utilizaram adequadamente o $\mathrm{DeCS}^{(2)}$.
Especificamente em Cirurgia Vascular e Angiologia cerca de 800 unitermos foram identificados nos DeCS ${ }^{3)}$.

\section{OBJETIVO}

Verificar a adequação dos unitermos utilizados por autores nacionais da especialidade de Cirurgia Vascular e Angiologia além de identificar modos de adequar a utilização e abrangência na padronização dos unitermos.

\section{MÉTODOS}

Foram pesquisadas duas revistas nacionais especializadas em temas vasculares, publicadas no período de 1995 a 2000.

As características de cada uma delas estão especificadas no quadro I. A denominação das revistas foi mantida em sigilo em resguardo de conflito de interesses.

QUADRO 1 - Características das duas revistas pesquisadas.

\begin{tabular}{l|c|c}
\hline & Revista A & Revista B \\
\hline Tiragem média & 2.000 & 2.000 \\
\hline Periodicidade & Trimestral & Trimestral \\
\hline Número de volumes & 16 & 9 \\
\hline Representatividade societária & Sim & Sim \\
\hline Número de editores & 5 & 4 \\
\hline Indexação na Medline & Não & Não \\
\hline Indexação na Lilacs & Sim & Sim \\
\hline Indexação na Scielo & Não & Não \\
\hline
\end{tabular}

Foram inicialmente listados todos os unitermos utilizados nos artigos publicados pelas duas revistas. A seguir esses termos foram confrontados com os unitermos existentes nos DeCS e com os unitermos listados por Timi et al. ${ }^{(3)}$ a partir do MeSH versão 1994.
A análise da confrontação procura verificar a freqüência dos termos citados nas duas listas ou em cada lista separadamente e os termos que não constaram de nenhuma das listas.

Considerando que a lista em língua inglesa (MeSH) tem mais abrangência foram consideradas as versões MeSH 2.000 e MeSH 1994. 
Oliveira, EFB de et al.

\section{RESULTADOS}

TABELA 1 - Freqüência dos descritores em língua inglesa utilizados em revistas brasileiras de angiologia e cirurgia vascular no período de 1995 a 2000.

( $\mathrm{NC}=$ não consta $/ \mathrm{C}=$ consta $)$

\begin{tabular}{|c|c|c|c|}
\hline Descritores & MeSH 2001 & MeSH 1994 & Numero de citações \\
\hline 1- Abdominal & $\mathrm{NC}$ & $\mathrm{NC}$ & 1 \\
\hline 2- Abdominal aorta & $\mathrm{NC}$ & $\mathrm{NC}$ & 5 \\
\hline 3- Abdominal aortic aneurysms & $\mathrm{NC}$ & $\mathrm{NC}$ & 4 \\
\hline 4- Abnormalities & $\mathrm{C}$ & $\mathrm{C}$ & 2 \\
\hline 5- Acute arterial occlusion & $\mathrm{NC}$ & $\mathrm{NC}$ & 2 \\
\hline 6- Ampicillin & $\mathrm{NC}$ & $\mathrm{NC}$ & 1 \\
\hline 7- Angiogenesis & $\mathrm{NC}$ & $\mathrm{NC}$ & 1 \\
\hline 8- Angiology and vascular surgery & $\mathrm{NC}$ & $\mathrm{NC}$ & 1 \\
\hline 9- Angioplasty & $\mathrm{C}$ & $\mathrm{C}$ & 5 \\
\hline 10- Anesthesia & $\mathrm{S}$ & $\mathrm{NC}$ & 1 \\
\hline 11- Anesthesia regional block & $\mathrm{NC}$ & $\mathrm{NC}$ & 1 \\
\hline 12- Aneurysm & $\mathrm{C}$ & $\mathrm{C}$ & 10 \\
\hline 13- Angiography & $\mathrm{C}$ & $\mathrm{C}$ & 3 \\
\hline 14- Angiology & $\mathrm{NC}$ & $\mathrm{NC}$ & 1 \\
\hline 15- Antibiotics & $\mathrm{C}$ & $\mathrm{C}$ & 1 \\
\hline 16- Antioxidants & $\mathrm{C}$ & $\mathrm{NC}$ & 1 \\
\hline 17- Antithrombin III & $\mathrm{C}$ & $\mathrm{C}$ & 1 \\
\hline 18- Aorta & $\mathrm{C}$ & $\mathrm{C}$ & 2 \\
\hline 19- Aortic & $\mathrm{NC}$ & $\mathrm{NC}$ & 1 \\
\hline 20- Aortic aneurysm & $\mathrm{C}$ & $\mathrm{C}$ & 4 \\
\hline 21- Aortic diseases & $\mathrm{C}$ & $\mathrm{C}$ & 2 \\
\hline 22- Aortic graft infection & $\mathrm{NC}$ & $\mathrm{NC}$ & 1 \\
\hline 23- Aortic sepsis & $\mathrm{NC}$ & $\mathrm{NC}$ & 1 \\
\hline 24- Aortoduodenal fistula & $\mathrm{NC}$ & $\mathrm{NC}$ & 1 \\
\hline 25- Aortoenteric fistula & $\mathrm{NC}$ & $\mathrm{NC}$ & 1 \\
\hline 26- Arterial catheters & $\mathrm{NC}$ & $\mathrm{NC}$ & 1 \\
\hline 27- Arterial bypass & $\mathrm{NC}$ & $\mathrm{NC}$ & 1 \\
\hline 28- Arterial embolism & $\mathrm{NC}$ & $\mathrm{NC}$ & 1 \\
\hline 29- Arterial occlusive diseases & $\mathrm{C}$ & $\mathrm{C}$ & 3 \\
\hline 30- Arterial occlusive diseases arteries & $\mathrm{C}$ & $\mathrm{C}$ & 1 \\
\hline 31- Arterial patch & $\mathrm{NC}$ & $\mathrm{NC}$ & 1 \\
\hline 32- Arterial plugs & $\mathrm{NC}$ & $\mathrm{NC}$ & 1 \\
\hline 33- Arterial prosthesis failure & $\mathrm{NC}$ & $\mathrm{NC}$ & 1 \\
\hline 34- Arterial surgery & $\mathrm{NC}$ & $\mathrm{NC}$ & 1 \\
\hline 35- Arteries & $\mathrm{C}$ & $\mathrm{C}$ & 2 \\
\hline 36- Arteriosclerosis & $\mathrm{C}$ & $\mathrm{C}$ & 1 \\
\hline 37- Arteriosclerotic vascular disease & $\mathrm{NC}$ & $\mathrm{NC}$ & 1 \\
\hline 38- Arteriovenous fistula & $\mathrm{C}$ & $\mathrm{C}$ & 3 \\
\hline 39- Arteriovenous shunt surgical & $\mathrm{NC}$ & $\mathrm{C}$ & 1 \\
\hline 40- Artery aneurysm & $\mathrm{NC}$ & $\mathrm{NC}$ & 2 \\
\hline 41- Artery canulation & $\mathrm{NC}$ & $\mathrm{NC}$ & 1 \\
\hline 42- Artery ruptured & $\mathrm{NC}$ & $\mathrm{NC}$ & 1 \\
\hline 43-Atherosclerosis & $\mathrm{C}$ & $\mathrm{C}$ & 6 \\
\hline 44- Atherosclerotic occlusive lesion & $\mathrm{NC}$ & $\mathrm{NC}$ & 1 \\
\hline 45- Atraumatic clamp & $\mathrm{NC}$ & $\mathrm{NC}$ & 1 \\
\hline 46- Bacterial infection & $\mathrm{NC}$ & $\mathrm{C}$ & 2 \\
\hline 47- Balloon angioplasty & $\mathrm{NC}$ & $\mathrm{NC}$ & 1 \\
\hline
\end{tabular}




\begin{tabular}{|c|c|c|c|}
\hline Descritores & MeSH 2001 & MeSH 1994 & Numero de citações \\
\hline 48- Ballon dilatation & $\mathrm{NC}$ & $\mathrm{C}$ & 1 \\
\hline 49- Basilar artery & $\mathrm{C}$ & $\mathrm{C}$ & 1 \\
\hline 50- Biomedical economic and social aspects & $\mathrm{NC}$ & $\mathrm{NC}$ & 1 \\
\hline 51- Blood flow & $\mathrm{NC}$ & $\mathrm{NC}$ & 1 \\
\hline 52- Blood vessels prosthesis & $\mathrm{NC}$ & $\mathrm{NC}$ & 1 \\
\hline 53- Bovine pericardium & $\mathrm{NC}$ & $\mathrm{NC}$ & 1 \\
\hline 54- Brachial artery & $\mathrm{NC}$ & $\mathrm{NC}$ & 2 \\
\hline 55- Brachytherapy & $\mathrm{C}$ & $\mathrm{NC}$ & 2 \\
\hline 56- Brain ischemia & $\mathrm{NC}$ & $\mathrm{NC}$ & 1 \\
\hline 57- Calf & $\mathrm{NC}$ & $\mathrm{NC}$ & 1 \\
\hline 58- Capillaries alterations & $\mathrm{NC}$ & $\mathrm{NC}$ & 1 \\
\hline 59- Capillaroscopy & $\mathrm{C}$ & $\mathrm{NC}$ & 1 \\
\hline 60- Cardiac catheterization & $\mathrm{NC}$ & $\mathrm{NC}$ & 1 \\
\hline 61- Carotid & $\mathrm{NC}$ & $\mathrm{NC}$ & 1 \\
\hline 62- Carotid surgery & $\mathrm{NC}$ & $\mathrm{NC}$ & 1 \\
\hline 63- Cath site & $\mathrm{NC}$ & $\mathrm{NC}$ & 1 \\
\hline 64- Catheters & $\mathrm{NC}$ & $\mathrm{NC}$ & 1 \\
\hline 65- Carodid artery & $\mathrm{NC}$ & $\mathrm{NC}$ & 7 \\
\hline 66- Carotid body tumor & $\mathrm{C}$ & $\mathrm{C}$ & 1 \\
\hline 67- Carotid endarterectomy & $\mathrm{C}$ & $\mathrm{NC}$ & 3 \\
\hline 68- Carotid vessels & $\mathrm{NC}$ & $\mathrm{NC}$ & 2 \\
\hline 69- Carotid stenosis & $\mathrm{C}$ & $\mathrm{C}$ & 6 \\
\hline 70- Cause of death & $\mathrm{C}$ & $\mathrm{C}$ & 1 \\
\hline 71- Cerebral aneurysms & $\mathrm{NC}$ & $\mathrm{C}$ & 1 \\
\hline 72- Cerebral angioplasty & $\mathrm{NC}$ & $\mathrm{NC}$ & 1 \\
\hline 73- Cerebral arteries & $\mathrm{C}$ & $\mathrm{C}$ & 2 \\
\hline 74- Cerebral ischemia & $\mathrm{C}$ & $\mathrm{C}$ & 3 \\
\hline 75- Cerebrovascular disorders & $\mathrm{C}$ & $\mathrm{C}$ & 3 \\
\hline 76- Claudication & $\mathrm{NC}$ & $\mathrm{NC}$ & 1 \\
\hline 77- Collateral circulation & $\mathrm{C}$ & $\mathrm{C}$ & 1 \\
\hline 78- Compartmental syndrome diagnosis & $\mathrm{NC}$ & $\mathrm{NC}$ & 1 \\
\hline 79- Complications of aneurisms & $\mathrm{NC}$ & $\mathrm{NC}$ & 1 \\
\hline 80- Contrast enhancement & $\mathrm{NC}$ & $\mathrm{NC}$ & 1 \\
\hline 81- Cosmetic treatment & $\mathrm{NC}$ & $\mathrm{NC}$ & 1 \\
\hline 82- Critical ischemia & $\mathrm{NC}$ & $\mathrm{NC}$ & 1 \\
\hline 83- Culture & $\mathrm{NC}$ & $\mathrm{NC}$ & 1 \\
\hline 84- Deep venous thrombosis & $\mathrm{NC}$ & $\mathrm{NC}$ & 4 \\
\hline $\begin{array}{l}\text { 85- Deep venous thrombosis antiplatelet } \\
\text { drugs prevention }\end{array}$ & $\mathrm{NC}$ & $\mathrm{NC}$ & 1 \\
\hline 86- Dermatofibrossarcoma & $\mathrm{NC}$ & $\mathrm{NC}$ & 1 \\
\hline 87- Descendig thoracic aorta & $\mathrm{NC}$ & $\mathrm{NC}$ & 1 \\
\hline 88- Diabetes & $\mathrm{NC}$ & $\mathrm{NC}$ & 1 \\
\hline 89- Diabetes mellitus & $\mathrm{C}$ & $\mathrm{C}$ & 2 \\
\hline 90- Diabetic angiopathy & $\mathrm{C}$ & $\mathrm{C}$ & 1 \\
\hline 91- Diabetic neuropathy & $\mathrm{NC}$ & $\mathrm{NC}$ & 1 \\
\hline 92- Diagnosis & $\mathrm{C}$ & $\mathrm{C}$ & 5 \\
\hline 93- Digital subtraction & $\mathrm{NC}$ & $\mathrm{NC}$ & 1 \\
\hline 94- Dipirone & $\mathrm{NC}$ & $\mathrm{NC}$ & 1 \\
\hline 95-Dissection & $\mathrm{C}$ & $\mathrm{C}$ & 3 \\
\hline 96- Distal bypass & $\mathrm{NC}$ & $\mathrm{NC}$ & 2 \\
\hline 97- Doppler & $\mathrm{NC}$ & $\mathrm{NC}$ & 1 \\
\hline 98- Doppler ultrasound & $\mathrm{C}$ & $\mathrm{NC}$ & 2 \\
\hline 99- Duplex scan & $\mathrm{NC}$ & $\mathrm{NC}$ & 8 \\
\hline
\end{tabular}




\begin{tabular}{|c|c|c|c|}
\hline Descritores & MeSH 2001 & MeSH 1994 & Numero de citações \\
\hline 100-Duplex ultrasound & $\mathrm{NC}$ & $\mathrm{NC}$ & 1 \\
\hline 101-Embolectomy & $\mathrm{C}$ & $\mathrm{NC}$ & 1 \\
\hline 102-Embolism & $\mathrm{C}$ & $\mathrm{NC}$ & 1 \\
\hline 103-Endoscopic surgery & $\mathrm{NC}$ & $\mathrm{NC}$ & 1 \\
\hline 104-Endothelium vascular & $\mathrm{NC}$ & $\mathrm{NC}$ & 1 \\
\hline 105-Endovascular neurosurgery & $\mathrm{NC}$ & $\mathrm{NC}$ & 1 \\
\hline 106-Endovascular treatament & $\mathrm{NC}$ & $\mathrm{NC}$ & 1 \\
\hline 107-Epidemiology & $\mathrm{C}$ & $\mathrm{NC}$ & 2 \\
\hline 108-Esthetic surgery & $\mathrm{C}$ & $\mathrm{NC}$ & 1 \\
\hline 109-Ethics & $\mathrm{C}$ & $\mathrm{C}$ & 1 \\
\hline 110-External pudendal & $\mathrm{NC}$ & $\mathrm{NC}$ & 1 \\
\hline 111-Extra-anatomic bypass & $\mathrm{NC}$ & $\mathrm{NC}$ & 1 \\
\hline 112-Extracorporeal circulation & $\mathrm{NC}$ & $\mathrm{NC}$ & 1 \\
\hline 113-Failing grafts & $\mathrm{NC}$ & $\mathrm{NC}$ & 1 \\
\hline 114-False aneurysm & $\mathrm{NC}$ & $\mathrm{NC}$ & 2 \\
\hline 115-Femoral vein & $\mathrm{C}$ & $\mathrm{C}$ & 1 \\
\hline 116-Fibromuscular displasia & $\mathrm{NC}$ & $\mathrm{NC}$ & 2 \\
\hline 117-Foot diseases & $\mathrm{C}$ & $\mathrm{C}$ & 1 \\
\hline 118-Free radicals & $\mathrm{NC}$ & $\mathrm{NC}$ & 1 \\
\hline 119-Graft surveillance & $\mathrm{NC}$ & $\mathrm{NC}$ & 1 \\
\hline 120-Genes & $\mathrm{C}$ & $\mathrm{NC}$ & 1 \\
\hline 121-Great saphenous & $\mathrm{NC}$ & $\mathrm{NC}$ & 1 \\
\hline 122-Growth factors & $\mathrm{NC}$ & $\mathrm{NC}$ & 1 \\
\hline 123-Hamartomas & $\mathrm{NC}$ & $\mathrm{NC}$ & 1 \\
\hline 124-Hand ischemia & $\mathrm{NC}$ & $\mathrm{NC}$ & 1 \\
\hline 125 -Hemodialysis & $\mathrm{C}$ & $\mathrm{C}$ & 2 \\
\hline 126-Heparin & $\mathrm{C}$ & $\mathrm{C}$ & 1 \\
\hline 127-Hemorrhage & $\mathrm{C}$ & $\mathrm{C}$ & 1 \\
\hline 128-Histology & $\mathrm{C}$ & $\mathrm{C}$ & 1 \\
\hline 129-Horseshoe Kidney & $\mathrm{NC}$ & $\mathrm{NC}$ & 1 \\
\hline 130-Hypercoagulable & $\mathrm{NC}$ & $\mathrm{NC}$ & 1 \\
\hline 131-Hypertension & $\mathrm{NC}$ & $\mathrm{NC}$ & 1 \\
\hline 132-Iatrogenic & $\mathrm{NC}$ & $\mathrm{NC}$ & 1 \\
\hline 133-In situ saphenus vein & $\mathrm{NC}$ & $\mathrm{NC}$ & 1 \\
\hline 134-In situ saphenous vein graft & $\mathrm{NC}$ & $\mathrm{NC}$ & 1 \\
\hline 135-Infected arterial graft & $\mathrm{NC}$ & $\mathrm{NC}$ & 1 \\
\hline 136-Infected aortic prothesis & $\mathrm{NC}$ & $\mathrm{NC}$ & 1 \\
\hline 137-Infection & $\mathrm{C}$ & $\mathrm{C}$ & 3 \\
\hline 138-Infra inguinal bypass & $\mathrm{NC}$ & $\mathrm{NC}$ & 1 \\
\hline 139-Infra inguinal revascularization & $\mathrm{NC}$ & $\mathrm{NC}$ & 2 \\
\hline 140-Inflamatory & $\mathrm{NC}$ & $\mathrm{NC}$ & 1 \\
\hline 141-Inflamatory aneurysm & $\mathrm{NC}$ & $\mathrm{NC}$ & 1 \\
\hline 142-Inflamatory aneurysms of the aorta & $\mathrm{NC}$ & $\mathrm{NC}$ & 1 \\
\hline 143-Inferior mesenteric artery & $\mathrm{NC}$ & $\mathrm{NC}$ & 1 \\
\hline 144-Injury & $\mathrm{NC}$ & $\mathrm{NC}$ & 1 \\
\hline 145-Innominate artery & $\mathrm{NC}$ & $\mathrm{NC}$ & 1 \\
\hline 146-Intermitent claudication & $\mathrm{NC}$ & $\mathrm{C}$ & 1 \\
\hline 147-Internal carotid artery & $\mathrm{NC}$ & $\mathrm{NC}$ & 1 \\
\hline 148-Internal iliac artery & $\mathrm{NC}$ & $\mathrm{NC}$ & 2 \\
\hline 149-Internal mammary artery & $\mathrm{NC}$ & $\mathrm{NC}$ & 1 \\
\hline 150-Irradiation & $\mathrm{NC}$ & $\mathrm{NC}$ & 2 \\
\hline 151-Insufficient perforating veins & $\mathrm{NC}$ & $\mathrm{NC}$ & 1 \\
\hline 152-Insuffiency & $\mathrm{NC}$ & $\mathrm{NC}$ & 1 \\
\hline
\end{tabular}




\begin{tabular}{|c|c|c|c|}
\hline Descritores & MeSH 2001 & MeSH 1994 & Numero de citações \\
\hline 153-Intermitent pneumatic compression & $\mathrm{NC}$ & $\mathrm{NC}$ & 1 \\
\hline 154-Intervencional neurorradiology & $\mathrm{NC}$ & $\mathrm{NC}$ & 1 \\
\hline 155-Intimal hyperplasia & $\mathrm{NC}$ & $\mathrm{NC}$ & 4 \\
\hline 156-Intraarterial infusion & $\mathrm{NC}$ & $\mathrm{NC}$ & 1 \\
\hline 157-Ischemia & $\mathrm{C}$ & $\mathrm{NC}$ & 4 \\
\hline 158-Ischemia-reperfusion & $\mathrm{NC}$ & $\mathrm{NC}$ & 1 \\
\hline 159-Kidney autologous transplantation & $\mathrm{NC}$ & $\mathrm{NC}$ & 1 \\
\hline 160-Kidney transpant & $\mathrm{NC}$ & $\mathrm{NC}$ & 1 \\
\hline 161-Lasers & $\mathrm{C}$ & $\mathrm{NC}$ & 1 \\
\hline 162-Lateral venorrhaphy & $\mathrm{NC}$ & $\mathrm{NC}$ & 1 \\
\hline 163-Left renal vein ligation & $\mathrm{NC}$ & $\mathrm{NC}$ & 1 \\
\hline 164-Low molecular weight heparin & $\mathrm{NC}$ & $\mathrm{NC}$ & 1 \\
\hline 165-Lower limbs & $\mathrm{NC}$ & $\mathrm{NC}$ & 1 \\
\hline 166-Lower limb revascularization & $\mathrm{NC}$ & $\mathrm{NC}$ & 1 \\
\hline 167-Lymphangitis & $\mathrm{C}$ & $\mathrm{C}$ & 1 \\
\hline 168-Lymphatic diseases & $\mathrm{C}$ & $\mathrm{C}$ & 2 \\
\hline 169-Lymphatic system & $\mathrm{C}$ & $\mathrm{C}$ & 3 \\
\hline 170-Lymphedema & $\mathrm{C}$ & $\mathrm{C}$ & 3 \\
\hline 171-Lymphocintilography & $\mathrm{NC}$ & $\mathrm{NC}$ & 2 \\
\hline 172-Macro-angiopathy & $\mathrm{NC}$ & $\mathrm{NC}$ & 1 \\
\hline 173-Magnetic resonace & $\mathrm{C}$ & $\mathrm{NC}$ & 1 \\
\hline 174-Management strategy & $\mathrm{NC}$ & $\mathrm{NC}$ & 1 \\
\hline 175-Malondialdehyde & $\mathrm{C}$ & $\mathrm{NC}$ & 1 \\
\hline 176-Mercury & $\mathrm{C}$ & $\mathrm{NC}$ & 1 \\
\hline 177-Microcircullation & $\mathrm{C}$ & $\mathrm{C}$ & 1 \\
\hline 178-Myocardial revascularization & $\mathrm{NC}$ & $\mathrm{NC}$ & 2 \\
\hline 179-Nitric oxide & $\mathrm{NC}$ & $\mathrm{C}$ & 1 \\
\hline 180 -Nom invasive vascular laboratory & $\mathrm{NC}$ & $\mathrm{NC}$ & 1 \\
\hline 181-Nuclear magnetic resonance & $\mathrm{NC}$ & $\mathrm{NC}$ & 1 \\
\hline 182-Occlusive arteriopathy & $\mathrm{NC}$ & $\mathrm{NC}$ & 1 \\
\hline 183-Occlusive disease & $\mathrm{NC}$ & $\mathrm{NC}$ & 1 \\
\hline 184-Ocupacional therapy & $\mathrm{NC}$ & $\mathrm{NC}$ & 1 \\
\hline 185-Operative surgery & $\mathrm{NC}$ & $\mathrm{NC}$ & 2 \\
\hline 186-Oxidative stress & $\mathrm{NC}$ & $\mathrm{NC}$ & 1 \\
\hline 187-Pathology & $\mathrm{C}$ & $\mathrm{NC}$ & 1 \\
\hline 188-Percutaneous angioplasty & $\mathrm{NC}$ & $\mathrm{NC}$ & 1 \\
\hline 189-Peripheral arterial aneurysms & $\mathrm{NC}$ & $\mathrm{NC}$ & 1 \\
\hline 190-Peripheral vascular disease & $\mathrm{NC}$ & $\mathrm{NC}$ & 1 \\
\hline 191-Phlebography & $\mathrm{C}$ & $\mathrm{C}$ & 4 \\
\hline 192-Photoplestimography & $\mathrm{NC}$ & $\mathrm{NC}$ & 2 \\
\hline 193-Plestysmography venous diagnosis & $\mathrm{NC}$ & $\mathrm{NC}$ & 1 \\
\hline 194-Politetrafluoroethylene & $\mathrm{NC}$ & $\mathrm{C}$ & 1 \\
\hline 195-Poplitea aneurysms & $\mathrm{NC}$ & $\mathrm{NC}$ & 1 \\
\hline 196-Popliteal artery & $\mathrm{C}$ & $\mathrm{C}$ & 3 \\
\hline 197-Popliteal vein & $\mathrm{C}$ & $\mathrm{C}$ & 1 \\
\hline 198-Popliteal venous aneurysms & $\mathrm{NC}$ & $\mathrm{NC}$ & 1 \\
\hline 199-Portal vein & $\mathrm{C}$ & $\mathrm{NC}$ & 1 \\
\hline 200-Pregmancy disease tratment & $\mathrm{NC}$ & $\mathrm{NC}$ & 1 \\
\hline 201-Primary aortoenteric fistula & $\mathrm{NC}$ & $\mathrm{NC}$ & 1 \\
\hline 202-Profunda femoral artery & $\mathrm{NC}$ & $\mathrm{NC}$ & 1 \\
\hline 203-Prostaglandins & $\mathrm{C}$ & $\mathrm{NC}$ & 1 \\
\hline 204-Prosthetic graft infection & $\mathrm{NC}$ & $\mathrm{NC}$ & 1 \\
\hline 205-Pseudoaneurysm & $\mathrm{C}$ & $\mathrm{NC}$ & 1 \\
\hline
\end{tabular}




\begin{tabular}{|c|c|c|c|}
\hline Descritores & MeSH 2001 & MeSH 1994 & Numero de citações \\
\hline 206-Pulmonary embolism & $\mathrm{NC}$ & $\mathrm{NC}$ & 1 \\
\hline 207-Rabbits & $\mathrm{C}$ & $\mathrm{NC}$ & 1 \\
\hline 208-Radial artery & $\mathrm{C}$ & $\mathrm{NC}$ & 1 \\
\hline 209-Radiation & $\mathrm{C}$ & $\mathrm{NC}$ & 2 \\
\hline 210-Radioisotopic & $\mathrm{NC}$ & $\mathrm{NC}$ & 1 \\
\hline 211-Rats & $\mathrm{C}$ & $\mathrm{NC}$ & 1 \\
\hline 212-Recurrent varicose veins & $\mathrm{NC}$ & $\mathrm{NC}$ & 1 \\
\hline 213-Recurrent veins & $\mathrm{NC}$ & $\mathrm{NC}$ & 1 \\
\hline 214-Restenosis & $\mathrm{C}$ & $\mathrm{NC}$ & 2 \\
\hline 215-Renal artery & $\mathrm{C}$ & $\mathrm{NC}$ & 2 \\
\hline 216-Renal artery angioplasty & $\mathrm{NC}$ & $\mathrm{NC}$ & 2 \\
\hline $217-$ Renal artery stenosis & $\mathrm{C}$ & $\mathrm{NC}$ & 2 \\
\hline 218-Renal artery obstrution & $\mathrm{NC}$ & $\mathrm{C}$ & 1 \\
\hline 219-Renal ischemia & $\mathrm{NC}$ & $\mathrm{NC}$ & 1 \\
\hline 220-Renal vein & $\mathrm{NC}$ & $\mathrm{C}$ & 2 \\
\hline 221-Renal transplantation & $\mathrm{NC}$ & $\mathrm{NC}$ & 1 \\
\hline 222-Renovascular hypertension & $\mathrm{NC}$ & $\mathrm{NC}$ & 5 \\
\hline 223-Retrograde femoral occlusion & $\mathrm{NC}$ & $\mathrm{NC}$ & 1 \\
\hline 224-Retroperitoneal fibrosis & $\mathrm{NC}$ & $\mathrm{NC}$ & 1 \\
\hline 225-Saphenectomy & $\mathrm{NC}$ & $\mathrm{NC}$ & 1 \\
\hline 226-Saphenous popliteal incompetence & $\mathrm{NC}$ & $\mathrm{NC}$ & 1 \\
\hline 227-Saphenous vein & $\mathrm{C}$ & $\mathrm{C}$ & 2 \\
\hline 228-Saphenous vein surgery & $\mathrm{NC}$ & $\mathrm{NC}$ & 1 \\
\hline 229-Sclerosis of telangiectasias & $\mathrm{NC}$ & $\mathrm{NC}$ & 1 \\
\hline 230-Sclerotherapy of varicose veins & $\mathrm{NC}$ & $\mathrm{NC}$ & 1 \\
\hline 231-Secondary aortoenteric fistula & $\mathrm{NC}$ & $\mathrm{NC}$ & 1 \\
\hline 232-Sinovial cyst & $\mathrm{NC}$ & $\mathrm{NC}$ & 1 \\
\hline 233-Short saphenous vein & $\mathrm{NC}$ & $\mathrm{NC}$ & 1 \\
\hline 234-Skin ulcer & $\mathrm{C}$ & $\mathrm{C}$ & 1 \\
\hline 235-Spleen & $\mathrm{C}$ & $\mathrm{NC}$ & 1 \\
\hline 236-Splenic & $\mathrm{C}$ & $\mathrm{NC}$ & 1 \\
\hline 237-Stent & $\mathrm{NC}$ & $\mathrm{NC}$ & 7 \\
\hline 238-Stent and protheses & $\mathrm{NC}$ & $\mathrm{NC}$ & 4 \\
\hline 239-Stent graft & $\mathrm{NC}$ & $\mathrm{NC}$ & 3 \\
\hline 240-Subclavian artery & $\mathrm{C}$ & $\mathrm{NC}$ & 4 \\
\hline 241-Subclavian artery steal syndrome & $\mathrm{NC}$ & $\mathrm{NC}$ & 1 \\
\hline 242-Subclavian vein puncture complication & $\mathrm{NC}$ & $\mathrm{NC}$ & 1 \\
\hline 243-Surgery & $\mathrm{C}$ & $\mathrm{C}$ & 12 \\
\hline 244-Surgical and clinical therapeutic aspects & $\mathrm{NC}$ & $\mathrm{NC}$ & 1 \\
\hline 245-Sural nerve & $\mathrm{C}$ & $\mathrm{C}$ & 1 \\
\hline 246-Surgery operative & $\mathrm{NC}$ & $\mathrm{C}$ & 1 \\
\hline 247-Surgical treatament & $\mathrm{NC}$ & $\mathrm{NC}$ & 1 \\
\hline 248-Surgical wound infection & $\mathrm{C}$ & $\mathrm{C}$ & 2 \\
\hline 249-Suture techiniques & $\mathrm{NC}$ & $\mathrm{C}$ & 1 \\
\hline 250-Sympathectomy & $\mathrm{NC}$ & $\mathrm{NC}$ & 1 \\
\hline 251-Tabagism & $\mathrm{NC}$ & $\mathrm{NC}$ & 1 \\
\hline 252-Takayasu's arteritis & $\mathrm{C}$ & $\mathrm{C}$ & 1 \\
\hline 253-Tangencial aneurysmectomy & $\mathrm{NC}$ & $\mathrm{NC}$ & 1 \\
\hline 254-Telangiectasis & $\mathrm{NC}$ & $\mathrm{NC}$ & 1 \\
\hline 255-Thoracic & $\mathrm{C}$ & $\mathrm{NC}$ & 1 \\
\hline 256-Thoracic aorta & $\mathrm{NC}$ & $\mathrm{NC}$ & 1 \\
\hline 257-Thrombectomy & $\mathrm{C}$ & $\mathrm{NC}$ & 2 \\
\hline 258-Thromboembolism & $\mathrm{C}$ & $\mathrm{C}$ & 2 \\
\hline 259-Thromboembolism venous & $\mathrm{NC}$ & $\mathrm{NC}$ & 1 \\
\hline
\end{tabular}




\begin{tabular}{|c|c|c|c|}
\hline Descritores & MeSH 2001 & MeSH 1994 & Numero de citações \\
\hline 260-Thrombophilia & $\mathrm{C}$ & $\mathrm{NC}$ & 1 \\
\hline 261-Thrombolysis & $\mathrm{C}$ & $\mathrm{NC}$ & 1 \\
\hline 262-Thrombosis treatment & $\mathrm{NC}$ & $\mathrm{NC}$ & 3 \\
\hline 263-Thrombosis venous & $\mathrm{NC}$ & $\mathrm{NC}$ & 5 \\
\hline 264-Transluminal angioplasty & $\mathrm{NC}$ & $\mathrm{NC}$ & 2 \\
\hline 265-Trauma & $\mathrm{C}$ & $\mathrm{NC}$ & 1 \\
\hline 266-Traumatic arteriovenous fistula & $\mathrm{NC}$ & $\mathrm{NC}$ & 2 \\
\hline 267-Treatment outcome & $\mathrm{C}$ & $\mathrm{NC}$ & 1 \\
\hline 268-Tuberculosis & $\mathrm{NC}$ & $\mathrm{NC}$ & 1 \\
\hline 269-Ulnar artery & $\mathrm{C}$ & $\mathrm{NC}$ & 1 \\
\hline 270-Ultrasound & $\mathrm{C}$ & $\mathrm{NC}$ & 1 \\
\hline 271-Ultrassonography & $\mathrm{NC}$ & $\mathrm{C}$ & 3 \\
\hline 272-Ultrassonography venous & $\mathrm{NC}$ & $\mathrm{NC}$ & 1 \\
\hline 273-Umbilical hérnia & $\mathrm{NC}$ & $\mathrm{NC}$ & 1 \\
\hline 274-Umbilical vein & $\mathrm{NC}$ & $\mathrm{C}$ & 1 \\
\hline 275-Uncle-brachial index & $\mathrm{NC}$ & $\mathrm{NC}$ & 1 \\
\hline 276-Varicose ulcer & $\mathrm{C}$ & $\mathrm{C}$ & 1 \\
\hline 277-Varicose veins & $\mathrm{C}$ & $\mathrm{C}$ & 14 \\
\hline 278-Varicose veins photoplesthysmography & $\mathrm{NC}$ & $\mathrm{NC}$ & 1 \\
\hline 279-Varicose veins of the lower limbs & $\mathrm{NC}$ & $\mathrm{NC}$ & 1 \\
\hline 280-Varicose veins recurrence & $\mathrm{NC}$ & $\mathrm{NC}$ & 1 \\
\hline 281-Varicose veins surgery & $\mathrm{NC}$ & $\mathrm{NC}$ & 1 \\
\hline 282-Varicose vein ultrassonography & $\mathrm{NC}$ & $\mathrm{NC}$ & 1 \\
\hline 283-Vascular anastomoses & $\mathrm{NC}$ & $\mathrm{NC}$ & 1 \\
\hline 284-Vascular iatrogenic & $\mathrm{NC}$ & $\mathrm{NC}$ & 1 \\
\hline 285-Vascular iatrogenic in children & $\mathrm{NC}$ & $\mathrm{NC}$ & 1 \\
\hline 286-Vascular iatrogenic injuries & $\mathrm{NC}$ & $\mathrm{NC}$ & 1 \\
\hline 287-Vascular graft extra anatomic & $\mathrm{NC}$ & $\mathrm{NC}$ & 1 \\
\hline 288-Vascular graft infection & $\mathrm{NC}$ & $\mathrm{NC}$ & 1 \\
\hline 289-Vascular graft occlusion & $\mathrm{NC}$ & $\mathrm{NC}$ & 1 \\
\hline 290-Vascular malformations & $\mathrm{NC}$ & $\mathrm{NC}$ & 1 \\
\hline 291-Vascular prothesis & $\mathrm{NC}$ & $\mathrm{NC}$ & 3 \\
\hline 292-Vascular surgery & $\mathrm{NC}$ & $\mathrm{NC}$ & 4 \\
\hline 293-Vascular trauma & $\mathrm{NC}$ & $\mathrm{NC}$ & 4 \\
\hline 294-Vascular trauma evolution & $\mathrm{NC}$ & $\mathrm{NC}$ & 1 \\
\hline 295-Vascular trauma in children & $\mathrm{NC}$ & $\mathrm{NC}$ & 1 \\
\hline 296-Vasodilatador drugs & $\mathrm{NC}$ & $\mathrm{NC}$ & 1 \\
\hline 297-Vasoespasm & $\mathrm{NC}$ & $\mathrm{C}$ & 1 \\
\hline 298-Vena cava inferior & $\mathrm{NC}$ & $\mathrm{NC}$ & 1 \\
\hline 299-Veins & $\mathrm{NC}$ & $\mathrm{C}$ & 5 \\
\hline 300-Veins acess & $\mathrm{NC}$ & $\mathrm{NC}$ & 1 \\
\hline 301-Venous & $\mathrm{C}$ & $\mathrm{C}$ & 1 \\
\hline 302-Venous catheterization & $\mathrm{NC}$ & $\mathrm{NC}$ & 1 \\
\hline 303-Venous insufficiency & $\mathrm{C}$ & $\mathrm{C}$ & 5 \\
\hline 304-Venous thromboembolic & $\mathrm{NC}$ & $\mathrm{NC}$ & 1 \\
\hline 305-Venous thromboembolism & $\mathrm{NC}$ & $\mathrm{NC}$ & 2 \\
\hline 306-Venous thromboembolism treatment & $\mathrm{NC}$ & $\mathrm{NC}$ & 1 \\
\hline 307-Venous thrombosis & $\mathrm{C}$ & $\mathrm{C}$ & 2 \\
\hline 306-Venous thrombosis prevention & $\mathrm{NC}$ & $\mathrm{NC}$ & 1 \\
\hline 309-Venous surgery & $\mathrm{NC}$ & $\mathrm{NC}$ & 1 \\
\hline 310 -Vertebral artery & $\mathrm{C}$ & $\mathrm{C}$ & 3 \\
\hline 311-Videoscopy & $\mathrm{NC}$ & $\mathrm{NC}$ & 1 \\
\hline 312-Visceral arterial aneurysms & $\mathrm{NC}$ & $\mathrm{NC}$ & 1 \\
\hline 313-Vitamin E & $\mathrm{C}$ & $\mathrm{C}$ & 1 \\
\hline
\end{tabular}


Oliveira, EFB de et al.

TABELA 2 - Freqüência absoluta e relativa dos descritores em língua inglesa usados em artigos de revistas nacionais de angiologia e cirurgia vascular no período de 1995 a 2000.

\begin{tabular}{lcc}
\hline \multicolumn{1}{c}{ Descritores } & Freqüência & Freqüência Relativa \\
\hline DeCS (2001) & 106 & $33,86 \%$ \\
MeSH (1994) & 79 & $25,24 \%$ \\
Ausentes & 176 & $56,23 \%$ \\
Presentes & 63 & $20,13 \%$ \\
Total & 313 & $100 \%$ \\
\hline
\end{tabular}

TABELA 3 - Freqüência absoluta e relativa dos descritores em língua inglesa citados mais de uma vez em artigos de revistas nacionais de angiologia e cirurgia vascular no período de 1995 a 2000.

\begin{tabular}{lcc}
\hline \multicolumn{1}{c}{ Descritores } & Freqüência & Freqüência Relativa \\
\hline DeCS (2001) & 45 & $40.17 \%$ \\
MeSH (1994) & 34 & $30,35 \%$ \\
Ausentes & 33 & $29,46 \%$ \\
Total & 112 & $100 \%$ \\
\hline
\end{tabular}

\section{DISCUSSÃO}

Visando confrontar os dados obtidos nesta pesquisa e os possíveis dados existentes na literatura, realizou-se uma busca nas bases de dados Lilacs e Medline no período de 1994 a 2001, usando o termo Descritor.

Encontrou-se quatro trabalhos, embora nenhum deles com a amostra e procedimentos iguais ao deste estudo.

Mesquita Júnior, Cepeda e Bella ${ }^{(5)}$ detectaram a ausência de uma base de dados que fosse útil para a especialidade médica de homeopatia e elaboraram um vocabulário homeopático específico que foi posteriormente incorporado pela Bireme.

Azevedo, Población e Goldenberg, ${ }^{(1)}$ estudaram 46 artigos publicados na Acta Cirúrgica Brasileira, com a intenção de analisar o grau de adequação das propostas de indexação dos autores e daquelas feitas pela Bireme e concluíram que: ${ }^{(1)}$ os autores não consultaram o DeCS com a intensidade que era desejável; ${ }^{(3)}$ o DeCS ofereceu terminologia médica adequada para a indexação, entretanto novos descritores mereciam ser incluídos, enquanto outros necessitavam revisão; (2) os indexadores da Bireme se ressentiam da falta de embasamento especializado na área biomédica; ${ }^{(4)}$ havia necessidade de os autores conhecerem melhor o DeCS e de os indexadores da Bireme terem uma acessória na área biomédica.

Lowe e Barnett ${ }^{(6)}$ publicaram artigo sobre a utilização do Medical Subject Headings (MeSH) na realização de buscas bibliográficas. Estes autores analisaram a estrutura e o uso do $\mathrm{MeSH}$, ressaltando de que maneira este vocabulário poderia auxiliar as dificuldades em pesquisas na Medline. Concluíram que a crescente importância desta base de dados e a tendência de os próprios usuários realizarem seus levantamentos bibliográficos tornavam crucial que os profissionais de saúde se familiarizassem com o MeSH.

No VI Congresso de Pneumologia e Tisiologia do Rio de Janeiro, Rosas e Guimarães (7) apresentaram pôster sobre a utilização do DeCS por autores de comunicações científicas de um periódico nacional - o Jornal de Pneumologia. Estes autores verificaram que nos fascículos publicados em 1996, não havia menção de descritores em $29 \%$ dos artigos e que a metade dos descritores empregados não se encontrava no DeCS.

Rosas ${ }^{(1)}$ estudou 29 dissertações de mestrado do curso de mestrado em Tisiologia e Pneumolologia do IDT-UFRJ e concluiu que: (1) $14 \%$ dos autores, das tese e dissertações do curso em tela (1990/1996), utilizam adequadamente o DeSC; (3) $47 \%$ dos descritores empregados, nas teses e dissertações do curso em tela 
são adequados segundo o DeCS; (2) O DeCS oferece termos médicos adequados para a indexação das teses e dissertações do curso em tela, pois apenas $6 \%$ dos descritores empregados e que não constam do DeCS, merecem ser incluídos em uma próxima edição.

A amostra desta pesquisa foi constituída pelos artigos publicados nas duas revistas especializadas em angiologia e cirurgia vascular no período de 1995 a 2000.

Dos 313 termos descritos dos artigos analisados $176(56,23 \%)$ não constavam da lista dos Descritores em Ciências da Saúde (DeCS) da Bireme ou dos termos agrupados do Medical Subject Headings (MeSH) por Timi et al. ${ }^{(3)}$. Considerando que o conhecimento do artigo científico em língua inglesa é ponto fundamental para a sua citação e ampliação de sua divulgação, verifica-se que as chances destes trabalhos estão bem diminuídas.

Já 106 termos $(33,86 \%)$ constavam do DeCS e 79 termos $(25,24 \%)$ constavam do $\mathrm{MeSH}$ agrupados por Timi et al. ${ }^{(3)}$.

Um total de 63 termos $(20,13 \%)$ constavam nas duas listagens analisadas.

Em relação aos termos citados mais de uma vez os termos que não constavam em nenhuma das duas listagens somaram $33(29,46 \%)$, os que constavam do DeCS somaram $45(40,17 \%)$ e os do MeSH somaram $34(30,35 \%)$.

\section{CONCLUSÕES}

1. A maioria dos descritores dos artigos em angiologia e cirurgia vascular $(56,23 \%)$, não constam da listagem dos Descritores em Ciências da Saúde da Bireme como seria desejável.

2. Novos termos que não constam na listagem dos Descritores em Ciências da Saúde da Bireme devem ser acrescentados em listagens futuras para acompanhar o desenvolvimento da especialidade.

3. As sociedades médicas devem incentivar seus autores a buscar os termos constantes na listagem dos Descritores em Ciências da Saúde para seus artigos e os indexadores da Bireme devem manter um intercâmbio de informações técnicas com estas sociedades de especialidades médicas e curso de pós-graduação na área.

\section{REFERÊNCIAS}

1. Azevedo JLMC, Población DA, Goldenberg S. Descritores nos artigos científicos. Acta Cir Bras 1990; 5(2):35-54.

2. Rosas P,Guimarães CA, Judice LF, Perreira CAC, Válio EBM Descritores em ciências da saúde nas teses e dissertações de mestrado na área de doenças respiratórias. Acta Cir Bras 1999;14(1):43-6

3. Timi JRR, Stanischesk IC, Abrão E. Unitermos de uso mais freqüente para o angiologista e cirurgião vascular. Angiol Cir Vasc 1994;10(3):104-9

4. DeCS. Descritores em Ciências da Saúde. 4.ed. São Paulo: BIREME/OPAS; 2001

5. Mesquita Jr A, Cepeda LMR, Bella S. Indexação bibliográfica computadorizada em homeopatia: uma revolução no estudo e na pesquisa. Rev Homeopatia 1990;55 (3):80-5

6. Lowe HJ, Barnett GO. Understanding and using the medical subject headings $(\mathrm{MeSH})$ vocabulary to perform literature searches. J Am Med Assoc 1994;271 (14):1103-8.

7. Rosas P, Guimarães CA. Descritores em ciências da saúde em periódico nacional. Rev Pulmão 1997; 6 (3):41. 
Oliveira EFB, Oliveira HB, Azevedo JLMC, Fagundes DJ. The current medical subjects headings in two brazilian vascular medical journals. Acta Cir Bras [serial online] 2003 Jan-Feb;18(1). Available from URL: http://www.scielo.br/acb.

ABSTRACT - MeSH - indexed internet health directories must provide a fast and safety research of the scientific information. Otherwise, for this purpose, it is necessary that the authors choose the adequate and available headings. Our research goal is to explore two brazilian vascular medical journals from the headings included in MEDLINE and LILACS documents and determine how well the authors were using the medical subject headings. The Medline and Lilacs database were searched from published 1996 to 2000 and queried with the list of DeCs 2001(MeSH Portuguese version) and $\mathrm{MeSH}$ 1994. We describe here that about 186 articles were reviewed, in two journals of the speciality and found that most of them were out of the standard terms suggested by the MeSH. We concluded, despite the indexation must be a dynamic performance, the authors must be better informed about the rules of inclusion of new headings and how to apply the adequate key words.

KEY WORDS - Journal article. Headings. Evaluation studies. Validation studies.

Conflito de interesse: nenhum

Correspondência:

Fonte de financiamento: nenhuma

Eymard Francisco Brito de Oliveira

Praça Henrique Batista, 10

37010-690 Varginha-MG

eymard@varginha.br

Data do recebimento: $15 / 08 / 2002$

Data da revisão: 11/09/2002

Data da aprovação: $21 / 11 / 2002$ 